\title{
CHAOS NEAR STRUCTURAL PHASE TRANSITION
}

\author{
H. Beige, M. Diestelhorst \\ Sektion Physik, Martin-Luther Universität Halle-Wittenberg \\ Friedemann-Bach-Platz 6, O-4020 Halle, Germany \\ R. Forster, J. Albers and J. Petersson \\ Fachbereich Physik, Universität des Saarlandes, 6600 Saarbrücken, Germany \\ (Received November 9, 1991)

\begin{abstract}
The studies of nonlinear dynamical dielectric properties of TGS which exhibits chaotic behaviour have been presented. The recorded phase portraits obtained from experiment are compared with the calculations based on different assumptions using a computer.
\end{abstract}

PACS numbers: 77.80.Bh

\section{Introduction}

The study of nonlinear dynamical systems which exhibit chaotic behaviour has been of considerable interest (e.g. [1-7]). The aim of the present paper is to show that the recording of the phase portrait of a series-resonance circuit, consisting of a TGS-crystal and a linear inductance, is useful for the study of the nonlinear dielectric properties of TGS above and below the phase transition.

A computer controlled measuring system recorded quantitatively the phase portrait of the resonance circuit and the hysteresis loop of the TGS-crystal at the sample by measuring the shift of the resonance frequency of the circuit in dependence on the driving field [8]. On the basis of different assumptions we calculated the phase portraits by means of a computer. The comparison of calculated and experimentally observed phase portraits gives us the opportunity to investigate the question, which piece of information provides the phase portrait.

\section{Experimental methods}

Figure 1 shows the equipment for recording the phase portrait of the series-resonance circuit. The measuring system is based on the fully programmable true dual-channel digitizing instrument Sony/Tektronix RTD 710. The digitizer provides 10 -bit resolution at a $200 \mathrm{MHz}$ maximum sampling rate (single-channel 


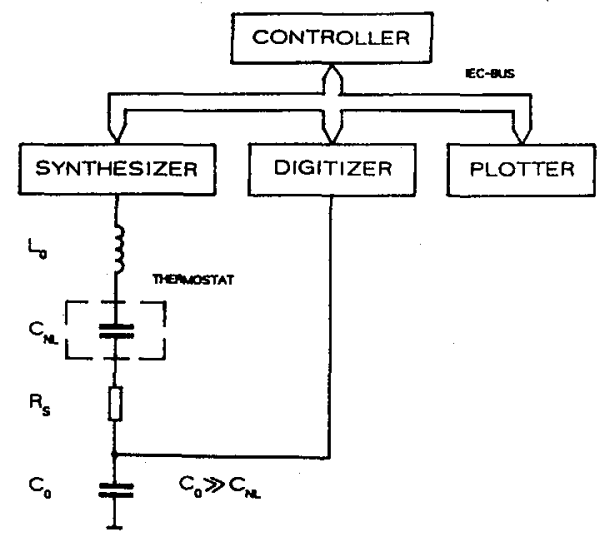

Fig. 1. Block diagram of the measuring system for recording the phase portrait.
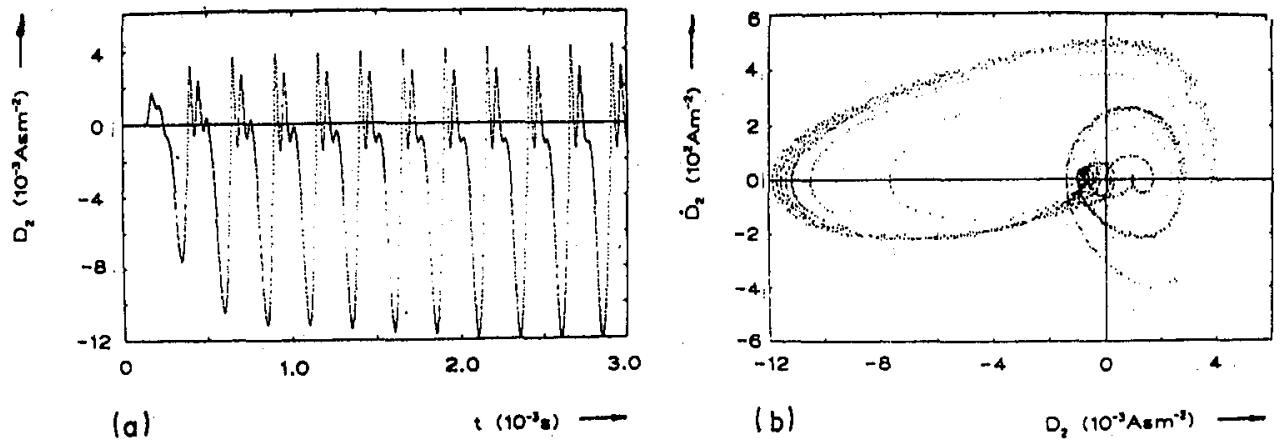

Fig. 2. Experimentally determined response function (a) and phase portrait of transient oscillations (b).

mode). The data memory is provided by $64 \mathrm{~K}$ words ( 10 bits per word) of local high-speed RAM. The synthesizer is fully programmable. All equipments are connected by a IEC-bus. An advantage of the computer controlled system is that the short measuring time reduces heating effects in the sample. The following notation is used: $C_{\mathrm{NL}}-$ the capacitance of the sample, $C_{0}-$ a linear capacitance, $R_{\mathrm{S}}$ - the loss of the resonance circuit and $L_{0}-$ a linear inductance.

The following differential equation describes the behaviour of the series-resonance circuit:

$$
\ddot{D}_{2}+\frac{\omega_{\mathrm{oe}}}{Q} \dot{D}_{2}+\frac{a}{b l L_{0}} E_{\mathrm{NL}}=\frac{U_{0}}{b l L_{0}} \cos \omega_{\mathrm{e}} t
$$

Here $D_{2}$ is the dielectric displacement, $a$ is the thickness of the sample, $l$ the length and $b$ the width. The electric field strength at the TGS-crystal along the ferroelectric b-axis is denoted by $E_{\mathrm{NL}}, U_{0}$ is the amplitude of the exciting voltage with the frequency $\omega_{\mathrm{e}}, Q$ denotes the quality of the resonance circuit and $\omega_{\text {oe }}$ 
the resonance frequency. Across the capacitance $C_{0}$ we obtain a signal proportional to $D_{2}$.

With the measuring system we can record the excitation $U_{0}(t)$, the response function, the phase portrait $\dot{D}_{2}=\dot{D}_{2}\left(D_{2}\right)$, where $\dot{D}_{2}$ is calculated by the computer, the Fourier spectra of the response function, a stroboscopic phase portrait and transient oscillations (Fig. 2). Removing the inductance $L_{0}$ (see Fig. 1) the hysteresis loop may be recorded.

\section{Nonlinear nature of the resonant system}

The ferroelectric phase transitions of second-order in TGS at $\vartheta_{\mathrm{c}}=49^{\circ} \mathrm{C}$ can be described by the thermodynamic potential:

$$
G=G_{0}+\frac{A}{2} D_{2}^{2}+\frac{B}{4} D_{2}^{4}
$$

The only temperature-dependent coefficient is $A=A_{0}\left(\vartheta-\vartheta_{c}\right)$. The electric field at the sample is calculated from Eq. (2) as:

$$
E_{\mathrm{NL}}=\frac{\partial G}{\partial D_{2}}=A D_{2}+B D_{2}^{3} \text {. }
$$

Using the relation (3) in Eq. (1) we obtain the so-called Duffing equation (e.g. [9]):

$$
\ddot{D}_{2}+\frac{\omega_{\mathrm{oe}}}{Q} \dot{D}_{2}+\frac{a A}{b l L_{0}} D_{2}+\frac{a B}{b l L_{0}} D_{2}^{3}=\frac{U_{0}}{b l L_{0}} \cos \omega_{\mathrm{e}} t .
$$

\section{Comparison of calculated and experimentally observed phase portraits}

We recorded the phase portraits of the series-resonance circuit at different temperatures, driving voltages and applied bias fields above and below the phase transition. Above the phase transition we used the shift of the resonance frequency $\Delta \omega_{\mathrm{ve}}$ of the resonance circuit as a function of the exciting voltage in order to determine the coefficients in Eq. (4). This relation is determined by the expression $[8,9]$ :

$$
\Delta \omega_{\mathrm{ve}}=\frac{3 Q^{2} \omega_{\mathrm{oe}} B}{16 a^{2} A^{3}} U_{0}^{2} .
$$

The calculated and experimentally observed phase portraits are in good agreement (see Figs. $3 \mathrm{~b}$ and $4 \mathrm{c}$ ).

Below the phase transition we used three different ways in order to determine the coefficients $A$ and $B$. Firstly we calculated the coefficients in the framework of the Landau theory from the coefficients of the high temperature phase. Secondly we used the shift of resonance frequency [6]:

$$
\Delta \omega_{\mathrm{ve}}=\frac{3 Q^{2} \omega_{\mathrm{oe}} B}{16 a^{2} A^{3}} U_{0}^{2} .
$$

In the third way we determined $A$ and $B$ using the values of spontaneous polarization $D_{2 \text { sp }}= \pm(-A / B)$ and the coercive field strength $E_{\text {coer }}=( \pm 2 A / 3)-(A / 3 B)$. The comparison of the calculated and experimentally observed phase portraits 


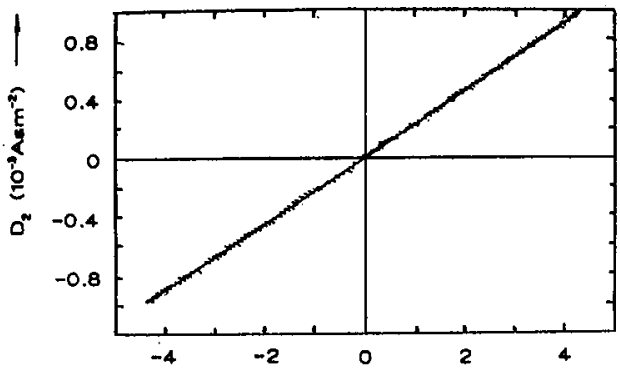

(a)

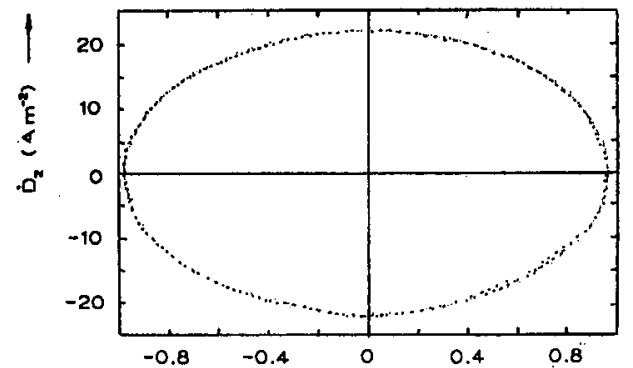

(b)
$D_{2}\left(10^{-3} \mathrm{Asm}^{-8}\right) \longrightarrow$

Fig. 3. Experimentally determined relation $D_{2}=D_{2}\left(E_{2}\right)$ (a) and phase portrait above $\vartheta_{\mathrm{c}}(\mathrm{b})$.

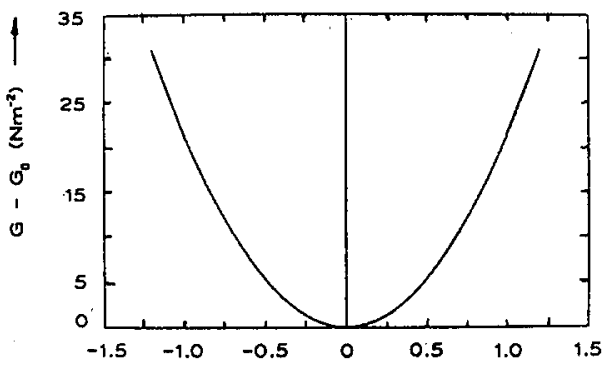

(a)

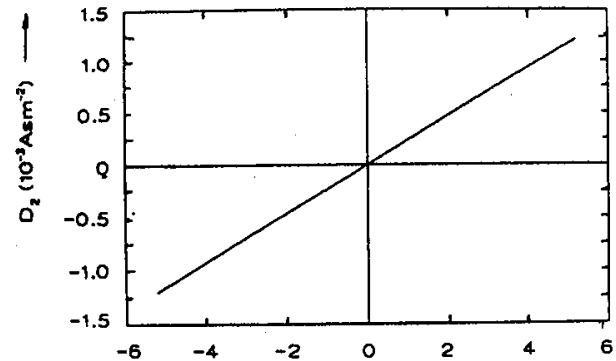

(b)

$E_{2}\left(10^{4} v m^{-1}\right) \longrightarrow$

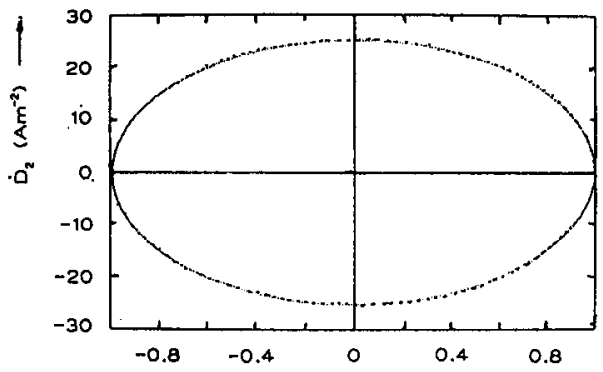

(c)

$D_{2}\left(10^{-3} \mathrm{Asm}^{-2}\right) \longrightarrow$

Fig. 4. Computer simulation of the thermodynamic potential (a), the relation $D_{2}=$ $D_{2}\left(E_{2}\right)$ (b) and the phase portrait above $\vartheta_{c}$ (c).

(see Figs. 5 and 6 ) provides only a good agreement with the values for the computer simulation derived form the hysteresis loop. (In the computer simulation we added the influence of an internal bias field.) Considering the measurements of the hysteresis loop and the phase portrait we note the influence for example of the domain reorientation on the nonlinear dielectric properties. This influence does 

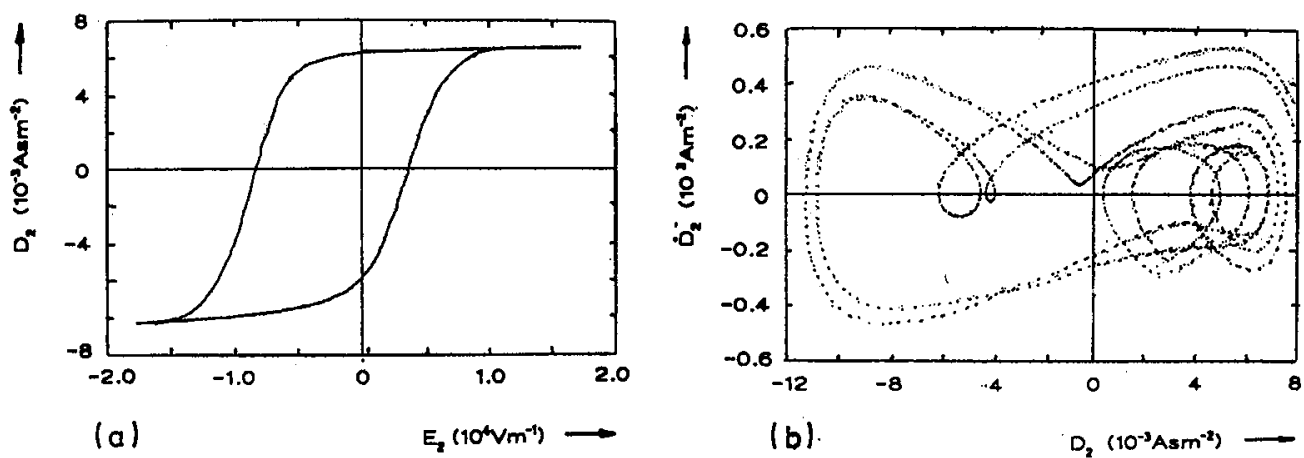

Fig. 5. Experimentally recorded hysteresis loop (a) and phase portrait below $\vartheta_{\mathrm{c}}(\vartheta=$ $\left.45.3^{\circ} \mathrm{C}\right)(\mathrm{b})$.

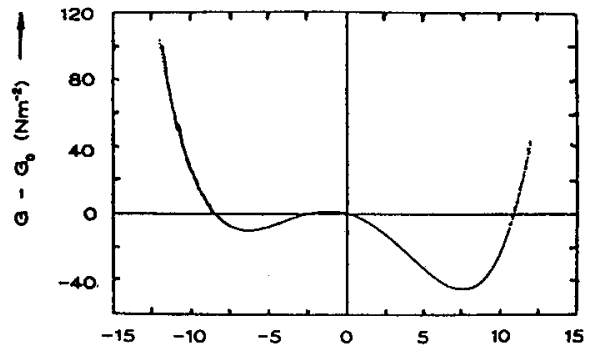

(a)

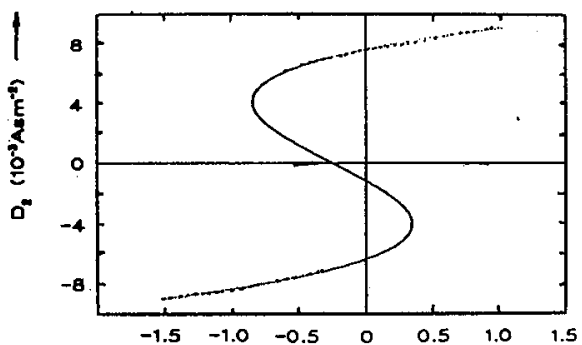

(b)

$E_{2}\left(10^{4} \mathrm{Vm}^{-1}\right) \longrightarrow$

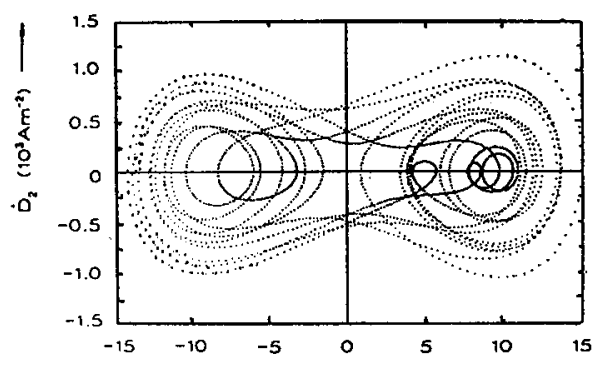

(c)

$\mathrm{D}_{2}\left(10^{-3} \mathrm{Asm} \mathrm{sm}^{-2}\right) \cdots$

Fig. 6. Computer simulation of the thermodynamic potential (a), the hysteresis loop (b) and the phase portrait below $\vartheta_{c}$ (c).

not contribute to measurements of the nonlinear dielectric coefficient by means of the shift of the resonance frequency because we work at amplitudes below the coercive field strength. Also in the Landau theory we do not take into consideration domain reorientation.

The conclusion that the phase portrait provides information about the "effective thermodynamic potential" is supported by the high sensitivity of the phase 
portrait against a bias field at the sample. Figure 7 represents the influence of very small bias fields $\left(E_{=}= \pm 2700 \mathrm{~V} / \mathrm{m}\right)$ on the portrait in the ferroelectric phase.
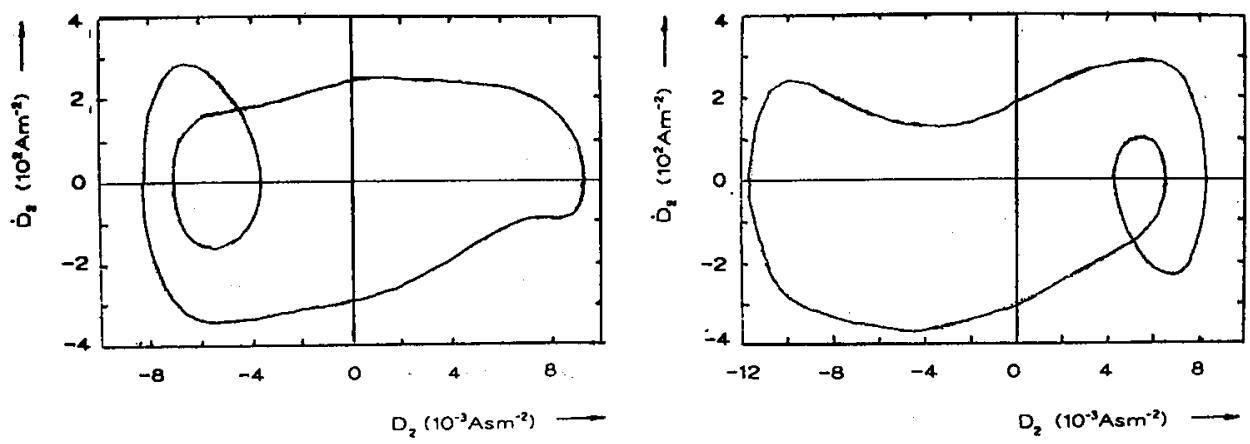

Fig. 7. Influence of the bias field on the phase portrait in the ferroelectric phase.

\section{Summary}

The experimentally recorded phase provides additional information about the nonlinear dynamic behaviour near the structural phase transition. With the knowledge of linear and nonlinear properties of the system we are able to calculate the phase portait using the computer. Particularly the comparison of calculated and experimentally recorded phase portraits yields hints about the nature of the nonlinear properties and acting physical mechanisms. Taking the phase portrait into account we obtain the opportunity to investigate the nonlinear dynamic in dependence on frequency in order to study for example switching processes in ferroelectric and ferroelastic materials.

An advantage of the measurements compared with numerical simulations is that we can identify the nonlinear phenomena with particular physical effects, that means in our case with dielectric nonlinearities in a ferroelectric crystal near the phase transition.

\section{References}

[1] D.J. Jeffries, Phys. Lett. A 90, 316 (1982).

[2] D.L. Gonzales, O. Piro, Phys. Rev. Lett. 50, 870 (1983).

[3] Y. Ueda, J. Stat. Phys. 20, 181 (1979).

[4] S. Martin, H. Leber, W. Martienssen, Phys. Rev. Lett. 53, 303 (1984).

[5] H. Beige, J. Albers, J. Peterson, Jpn. J. Appl. Phys. Suppl. 24-2, 715 (1985).

[6] M. Diestelhorst, Ferroelektrizität 87, WB MLU 67 (024) 68 (1987).

[7] M. Diestelhorst, H. Beige, Ferroelectrics 81, 979 (1988).

[8] H. Beige, G. Schmidt, Ferroelectrics 41, 173 (1982).

[9] A.H. Nayfeh, D.T. Mock, Nonlinear Oscillations, Wiley-Interscience, New York 1979. 